Revista Eletrônica de Ciência Administrativa (RECADM) - ISSN 1677-7387

Faculdade Cenecista de Campo Largo - Coordenação do Curso de Administração

v. 2, n. 1, maio/2003 - http://revistas.facecla.com.br/index.php/recadm/

\title{
PRONÓSTICO DE VENTAS: COMPARACIÓN DE PREDICCIÓN ENTRE REDES NEURONALES Y MÉTODO ESTADÍSTICO
}

\author{
Nojek, S. ${ }^{1}$, Britos, P. ${ }^{1,2}$, Rossi, B. ${ }^{1,2}$ y García Martínez, R. ${ }^{2}$ \\ ${ }^{1}$ Departamento de Ingeniería Industrial. Instituto Tecnológico de Buenos Aires. Av. Madero 399 \\ (C1106ACD). Buenos Aires - Argentina. \\ sebasnojek@fibertel.com.ar \\ ${ }^{2}$ Centro de Ingeniería \\ del Software e Ingeniería del Conocimiento (CAPIS). Instituto Tecnológico de Buenos Aires. Av. \\ Madero 399 (C1106ACD). Buenos Aires - Argentina. \\ pbritos@itba.edu.ar, brossi@itba.edu.ar, rgm@itba.edu.ar
}

Resumo: $\mathrm{Na}$ atualidade as empresas realizan pronostico da ventas pra poder façer uma mais ajustada planificaçao da suas produçoes. El objetivo da pesquisa descrita em este artigo es estudo das redes neurais nel pronóstico da ventas, contrastando os resultados obtidos con los producidos por um método estadístico clásico.

Palavras-Chave: Pronóstico da Ventas - Pronóstico basado em Redes Neurais

Resumen: Actualmente es común que las empresas realicen pronósticos de ventas para poder planificar más acertadamente su producción. El objetivo de este trabajo consiste en estudiar la predicción de ventas mediante el uso de redes neuronales, comparar los resultados obtenidos con los pronósticos de un método estadístico clásico y establecer los entornos más adecuados para su uso.

Palabras Clave: Pronostico de Ventas - Predicción basada en Redes Neuronales

Abstract: Companies usually need sales forecast for improving production planning. This research work pursues to study sales forecast based on neural networks and to compare this results whith the ones based on classical statistical methods trying to specify the adecuate bussines enviroment for using them

Keywords: Sales Forecast - Forcasting based on Neural Networks

\section{Introducción:}

Actualmente es común que las empresas realicen pronósticos de ventas para poder planificar más acertadamente su producción. El objetivo de este trabajo consiste en estudiar la predicción de ventas mediante el uso de redes neuronales, comparar los resultados obtenidos con los pronósticos de un método estadístico clásico y establecer los entornos más adecuados para su uso. 
Revista Eletrônica de Ciência Administrativa (RECADM) - ISSN 1677-7387

Faculdade Cenecista de Campo Largo - Coordenação do Curso de Administração

v. 2, n. 1, maio/2003 - http://revistas.facecla.com.br/index.php/recadm/

\section{Situación actual:}

La elección e implementación de un método adecuado de pronósticos siempre ha sido un tema de gran importancia para las empresas. Se utilizan los pronósticos en el área de compras, marketing, ventas, etc. Un error significante en el pronóstico de ventas podría dejar a una empresa sin la materia prima o insumos necesarios para su producción, o podría generarle un inventario demasiado grande. En ambos casos, el pronóstico erróneo disminuye las utilidades de la empresa. Las consecuencias anteriormente descriptas se ven amplificadas y representan un problema grave para empresas como Shell CAPSA (Compañía Argentina de Petróleo S. A.) en las que, según Guillermo Lucero, quien trabaja en la producción y realiza el planeamiento de la distribución de los productos de esta empresa para todo Latinoamérica, se debe realizar mensualmente un pronóstico para más de mil productos.

La estimación del comportamiento futuro de algunas variables puede realizarse utilizando diversas técnicas de pronóstico. Cada una de las técnicas de proyección tiene una aplicación de carácter especial que hace de su selección un problema de decisión influido por diversos factores, como por ejemplo, la validez y disponibilidad de los datos históricos, la precisión deseada del pronóstico, el costo del procedimiento, los beneficios del resultado, los periodos futuros que se desee pronosticar y el tiempo disponible para hacer el estudio entre otros [Sapag, 2000].

En una situación estable la importancia de los pronósticos es menor. Pero a medida que ella crece en dinamismo y complejidad, más necesaria se torna la proyección de las variables del mercado.

Como se mencionó anteriormente, existen diversas técnicas para proyectar el mercado. Dentro de las mismas se encuentran las técnicas clásicas de proyección. Una forma de clasificarlas consiste en hacerlo en función de su carácter, esto es, aplicando métodos de carácter cualitativo, modelos causales y modelos de series de tiempo.

- Los métodos de carácter cualitativo se basan principalmente en opiniones de expertos. Su uso es frecuente cuando el tiempo para elaborar el pronóstico es escaso, cuando no se dispone de todos los antecedentes mínimos necesarios o cuando los datos disponibles no son confiables para predecir el comportamiento futuro. Resulta difícil emitir un juicio sobre la eficacia de sus estimaciones finales.

- Los modelos de pronóstico causales parten del supuesto de que el grado de influencia de las variables que afectan al comportamiento del mercado permanece estable, para luego construir un modelo que relacione ese comportamiento con las variables que se estima que son las causantes de los cambios que se observan en el mercado [Dervitsiotis, 1981]. 
Revista Eletrônica de Ciência Administrativa (RECADM) - ISSN 1677-7387

Faculdade Cenecista de Campo Largo - Coordenação do Curso de Administração

v. 2, n. 1, maio/2003 - http://revistas.facecla.com.br/index.php/recadm/

- Los modelos de series de tiempo (método estadístico) se refieren a la medición de valores de una variable en el tiempo a intervalos espaciados uniformemente. El objetivo de la identificación de la información histórica es determinar un patrón básico en su comportamiento, que posibilite la proyección futura de la variable deseada.

Existen, además de los métodos clásicos de proyección, diversos métodos de proyección no tradicionales, dentro de los cuales se encuentran las redes neuronales. Debido a su constitución y a sus fundamentos, las redes neuronales artificiales presentan un gran número de características semejantes a las del cerebro humano. Por ejemplo, son capaces de aprender de la experiencia, de generalizar de casos anteriores a nuevos casos y de abstraer características esenciales a partir de entradas que representan información irrelevante [Hilera y Martínez,1995].

\section{Hipótesis de la investigación:}

Hipótesis: Utilizando un método de pronóstico no tradicional, como las redes neuronales, es posible obtener un pronóstico de ventas más acertado en comparación a los resultados obtenidos utilizando un método de pronóstico estadístico clásico.

La metodología propuesta para este trabajo fue la siguiente:

1. Obtener los datos históricos de ventas de algunos productos de la empresa Shell CAPSA.

2. Realizar un pronóstico de ventas utilizando distintos métodos estadísticos. Para este trabajo se va a utilizar un software de la empresa J. D. Edwards, el Supplier Relationship Management [SRM, 1996], el cual incluye 12 (doce) métodos estadísticos distintos.

3. Utilizando los mismos datos de entrada que se utilizaron para realizar el pronóstico estadístico, se va a realizar un pronóstico utilizando redes neuronales. Se va a utilizar el software de la empresa Statsoft en Neural Network.

4. Comparar los resultados obtenidos por ambos métodos.

5. Definir el nivel de confianza (de 0 a $100 \%$ ) con el cual se puede decir que con las Redes Neuronales se obtuvo, para la muestra de datos previamente seleccionada, un pronóstico más acertado.

\section{Descripción de los métodos utilizados:}

\subsection{Método estadístico:}

En la actualidad existen productos software que integran varios métodos de pronóstico estadísticos. Para este trabajo, como se menciono anteriormente, se utilizó un software de la empresa J. D. Edwards, el Supplier Relationship Management (SRM). El mismo utiliza doce métodos de pronóstico estadísticos distintos. 
Revista Eletrônica de Ciência Administrativa (RECADM) - ISSN 1677-7387

Faculdade Cenecista de Campo Largo - Coordenação do Curso de Administração

v. 2, n. 1, maio/2003 - http://revistas.facecla.com.br/index.php/recadm/

El sistema de calculo del software, según la información que nos brinda el manual del mismo, funciona de la siguiente manera: primero, el sistema determina cuál de los 12 métodos se ajusta mejor a los datos proporcionados por el usuario (datos históricos) y luego, el sistema utiliza el método seleccionado para realizar las proyecciones de ventas.

Los 12 métodos utilizados fueron:

Método 1: Porcentaje sobre las ventas del año anterior.

La fórmula Porcentaje sobre las ventas del año anterior multiplica los datos del año anterior por un porcentaje determinado por el usuario, luego proyecta los resultados para el año siguiente.

Ejemplo: $\quad$ Ventas Febrero $1998=$ Ventas Febrero $1997 * 1.1$

Ventas Marzo $1998=$ Ventas Marzo $1997 * 1.1$

Método 2: Porcentaje sobre el año pasado calculado.

La fórmula Porcentaje sobre el año pasado calculado multiplica los datos del año anterior por un porcentaje determinado por el sistema, luego proyecta los resultados para el año siguiente.

Método 3: Ventas del año anterior.

La fórmula Ventas del año anterior copia los valores de las ventas del año anterior.

Método 4: Promedio móvil .

Este método calcula el promedio de las ventas históricas más recientes y realiza un pronóstico a corto plazo.

Método 5: Aproximación lineal.

Realiza una aproximación lineal en base a dos datos de ventas históricos determinados por el usuario. Estos puntos definen una línea recta que es proyectada hacia el futuro.

Método 6: Regresión de cuadrados mínimos.

El método calcula automaticamente los valores de "a" $y$ "b" que se utilizan en la fórmula: $Y=a+b X$. Esta ecuación describe una línea recta en donde $Y$ representa las ventas y $X$ representa al eje temporal.

Método 7: Aproximación de segundo grado.

El método calcula automaticamente los valores de "a", "b" y "c" que se utilizan en la ecuación de segundo grado, $Y=a+b X+c X^{2}$. El eje $Y$ representa las ventas y el eje $X$ representa al eje temporal.

\section{Método 8: Método flexible.}

Este método es similar al método 1 (Porcentaje sobre el año pasado). El usuario determina un factor especifico, el cual se multiplica por las ventas de un periodo determinado (también por el usuario).

\section{Método 9: Promedio móvil ponderado.}

Este método es similar al método 4 (Promedio móvil). El usuario debe determinar: $n$, numero de periodos a tener en cuenta, y el peso que se le asigna a cada uno de los periodos pasados.

\section{Método 10: Decaimiento lineal.}

Este método es similar al método 9. La principal diferencia esta el peso que relativo que se le asigna a cada periodo lo determina el sistema automaticamente.

\section{Método 11: Decaimiento exponencial.}

Este método es similar al método 10. El sistema le asigna a cada peso relativo valores que decaen exponencialmente. El usuario debe determinar dos factores: alfa, constante de decaimiento, y $n$, el numero de datos pasados a tener en cuenta en cada pronóstico.

\section{Método 12: Decaimiento exponencial con tendencia y estacionalidad.}

Es similar al método anterior. Se diferencia principalmente porque incluye además un factor de estacionalidad beta. 
Revista Eletrônica de Ciência Administrativa (RECADM) - ISSN 1677-7387

Faculdade Cenecista de Campo Largo - Coordenação do Curso de Administração

v. 2, n. 1, maio/2003 - http://revistas.facecla.com.br/index.php/recadm/

\subsection{Redes Neuronales:}

Para este trabajo se propuso utilizar un software de redes neuronales con el fin de realizar los pronósticos de ventas. El software utilizado fue el Neural Neutwork de la empresa Statsoft.

Algunas de las preguntas que surgen como consecuencia del enfoque estadístico actualmente utilizado y que se intentaron responder utilizando las redes neuronales fueron las siguientes:

- ¿Existe un método de predicción que bajo determinadas condiciones realice pronósticos más precisos que el método estadístico?

- ¿Qué conocimientos se necesitan para realizar un pronóstico estadístico? ¿Y para realizar un pronóstico utilizando Redes Neuronales?

Las redes neuronales son sistemas dinámicos autoadaptativos. Son adaptables debido a la capacidad de autoajustarse de los elementos procesales (neuronal) que componen el sistema. Son dinámicos, pues son capaces de estar constantemente cambiando para adaptarse a las nuevas condiciones [Hilera y Martínez, 1995].

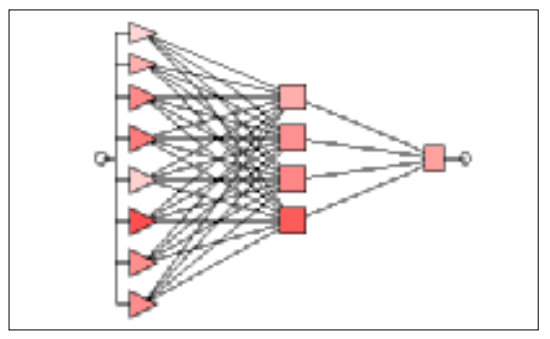

Fig. 1 - Ejemplo de Red neuronal

En el proceso de aprendizaje, los enlaces ponderados de las neuronas se ajustan de manera que se obtengan unos resultados específicos. Una red neuronal no necesita un algoritmo para resolver un problema, ya que ella puede generar su propia distribución de los pesos de los enlaces mediante el aprendizaje.

En este trabajo, se utilizó una red neuronal backpropagation para predecir la variable ventas debido a que esta red es especialmente útil en aplicaciones de reconocimiento o clasificación de patrones. En este modelo de red existe una capa de entrada con $n$ neuronas y una capa de salida con $m$ neuronas y al menos una capa oculta de neuronas internas. Cada neurona de una capa (excepto las de entrada) recibe entradas de todas las neuronas de la capa anterior y envía su salida a todas las neuronas de la capa posterior (excepto las de salida). 
Revista Eletrônica de Ciência Administrativa (RECADM) - ISSN 1677-7387

Faculdade Cenecista de Campo Largo - Coordenação do Curso de Administração

v. 2, n. 1, maio/2003 - http://revistas.facecla.com.br/index.php/recadm/

El algoritmo backpropagation tiene dos fases, una hacia delante y otra hacia atrás. Durante la primera fase el patrón de entrada es presentado a la red y propagado a través de las capas hasta llegar a la capa de salida. Obtenidos los valores de salida de la red, se inicia la segunda fase, comparándose estos valores con la salida esperada para obtener el error. Se ajustan los peses de la ultima capa proporcionalmente al error. Se pasa a la capa anterior con una retropropagación del error (backpropagation), ajustando convenientemente los pesos y continuando con este proceso hasta llegar a la primera capa. De esta manera se han modificado los pesos de las conexiones de la red para cada ejemplo o patrón de aprendizaje del problema, del que conocíamos su valor de entrada y la salida deseada que debería generar la red ante dicho patrón.

\subsubsection{Descripción de las redes utilizadas:}

Existen cuatro aspectos que caracterizan una red neuronal: su topología, el mecanismo de aprendizaje, tipo de asociación realizada entre la información de entrada y salida, y por ultimo, la forma de representación de estas informaciones [Hilera y Martinez, 1995]. En este trabajo, se estudiaron los resultados obtenidos luego de utilizar cuatro configuraciones de red distintas.

\section{Topología de las redes utilizadas:}

Como se adelantó anteriormente, se utilizaron cuatro configuraciones o arquitecturas de redes neuronales. Se realizaron pronósticos con las cuatro topologías. Estas pruebas se denominaron: prueba 1, prueba 2 , prueba 3 y prueba 4 respectivamente.

Tipo de conexiones (hacia delante): En las redes con conexiones hacia delante, todas las señales neuronales se propagan hacia delante a través de las capas de la red. No existen conexiones hacia atrás y normalmente tampoco autorrecurrentes (salida de una neurona aplicada a su propia entrada), ni laterales.

Numero de capas : 3 en todas las pruebas. Entrada, oculta y salida.
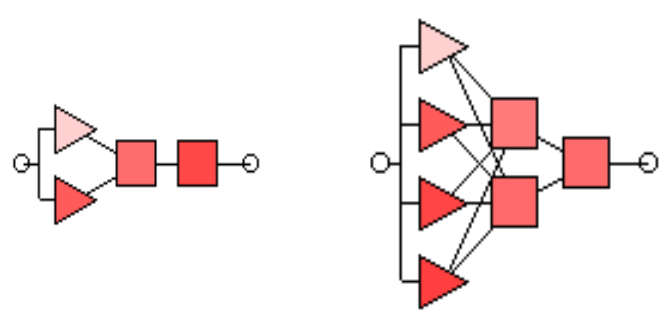

Fig. 2 - Topología I
Fig. 3 - Topología I

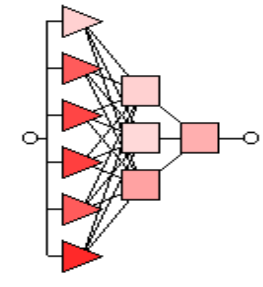

Fig. 4 - Topología III

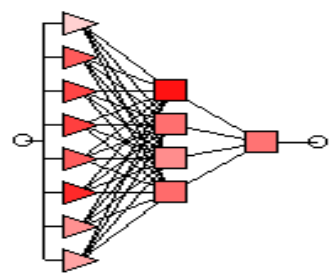

Fig. 5 - Topología IV

La capa de entrada recibe directamente la información proveniente de las fuentes externas. En este caso recibe los datos históricos de ventas. Las capas ocultas son internas de la red y no tienen contacto directo con el entorno exterior. Las capas de salida transfieren la información de la red hacia el exterior. 
Revista Eletrônica de Ciência Administrativa (RECADM) - ISSN 1677-7387

Faculdade Cenecista de Campo Largo - Coordenação do Curso de Administração

v. 2, n. 1, maio/2003 - http://revistas.facecla.com.br/index.php/recadm/

\section{Numero de neuronas por capa:}

\begin{tabular}{|c|c|c|c|c|}
\hline & \multicolumn{4}{|c|}{ Topología } \\
\hline & I & II & III & IV \\
\hline Entrada & 1 & 1 & 1 & 1 \\
\hline 의 Oculta & 1 & 2 & 3 & 4 \\
\hline Salida & 1 & 1 & 1 & 1 \\
\hline Steps & 2 & 4 & 6 & 8 \\
\hline
\end{tabular}

\section{Cuadro 1 - Neuronas por Topología}

Step: Es el número de datos pasados que la red tomo como entrada. Por lo tanto, la entrada a la red está formada por una neurona (una variable), pero según varíe su topología, tendrá en cuenta distinta cantidad de datos históricos simultáneamente. Por ejemplo, para la topología II (steps =4), cuando el dato de las ventas de la semana $n$ se encuentra en la capa de salida, las ventas de las semanas n-1, n-2, n-3 y n-4 se encuentran en la capa de entrada.

\section{Mecanismo de aprendizaje:}

El aprendizaje es el proceso por el cual una red neuronal modifica sus pesos en respuesta a una información de entrada. Los cambios que se producen durante el proceso de aprendizaje se reducen a la destrucción, modificación y creación de conexiones entre las neuronas.

En todas las pruebas realizadas se utilizó el tipo de aprendizaje denominado aprendizaje supervisado. El mismo se caracteriza porque el proceso de aprendizaje se realiza mediante un entrenamiento controlado por un agente externo (supervisor) que determina la respuesta que debería generar la red a partir de una entrada determinada.

\section{Tipo de información de entrada y salida:}

Forma de representar la información de entrada y salida: En todas las pruebas, tanto los datos de entrada como de salida fueron de naturaleza analógica; es decir que las ventas se tomaron en todos los casos como valores reales continuos. 
Revista Eletrônica de Ciência Administrativa (RECADM) - ISSN 1677-7387

Faculdade Cenecista de Campo Largo - Coordenação do Curso de Administração

v. 2, n. 1, maio/2003 - http://revistas.facecla.com.br/index.php/recadm/

\subsubsection{Entrenamiento de la red neuronal:}

Luego de crear la red neuronal se debe entrenar a la misma. Se debe entender por el término entrenar, la presentación de los datos históricos a la red neuronal. Para entrenar adecuadamente a las redes neuronales se deben ajustar algunos parámetros:

Fase I: Especificar qué datos históricos se utilizaran para entrenar a la red y cuáles se utilizarán para verificar (supervisar) que el entrenamiento haya finalizado o llegado al punto deseado.

Fase II: Determinar cuando debe detenerse el entrenamiento de la red. En todos los casos se determinó un error target del $0.1 \%$.

Fase III: Verificar gráficamente que los datos utilizados para entrenar a la red se ajusten gráficamente a los datos históricos de ventas.

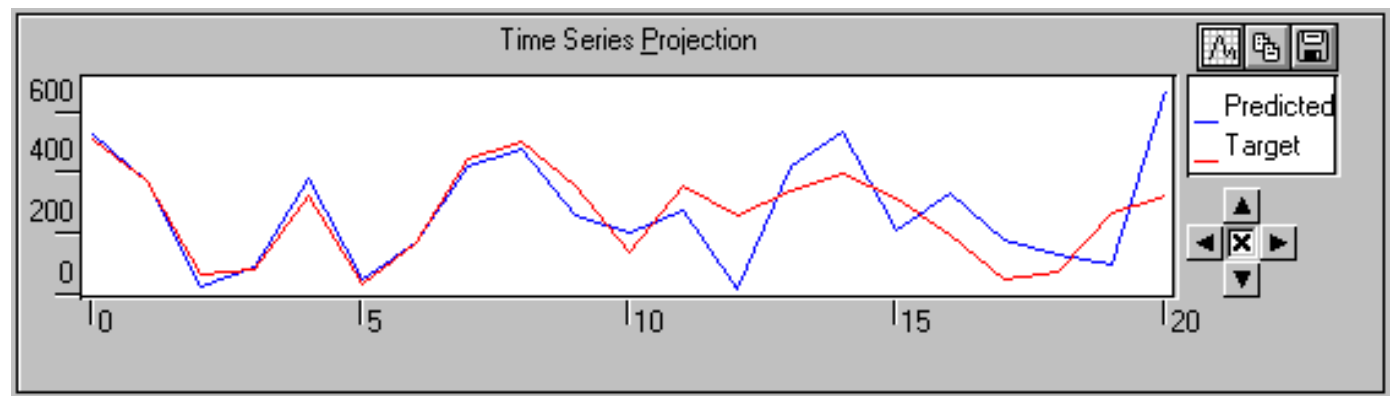

Fig. 6 - Ejemplo en el software utilizado

Fase IV: Obtener el pronóstico de Ventas.

\section{Experimentación:}

\subsection{Descripción de los datos utilizados:}

Los datos utilizados en este trabajo fueron provistos por Guillermo Lucero, quien planifica para Shell CAPSA la distribución de sus productos. Los mismos corresponden a las ventas de siete productos para los meses de Noviembre y Diciembre del 2001, Enero, Febrero, Marzo, Abril, Mayo y Junio del año 2002 (Apéndice A).

Se estudiaron siete series de tiempo. Cada una de las cuales pertenece a un producto de la empresa Shell CAPSA. Por una cuestión de confidencialidad se prefirió no publicar los nombres reales de los productos. Las series de tiempo fueron denominadas serie $1,2,3, \ldots .$. . 7 respectivamente (ver apéndice A). Cada una de estas series presentó en el pasado patrones de comportamiento distintos (productos con demandas estacionales, estables, aleatorios, etc.). 
Revista Eletrônica de Ciência Administrativa (RECADM) - ISSN 1677-7387

Faculdade Cenecista de Campo Largo - Coordenação do Curso de Administração

v. 2, n. 1, maio/2003 - http://revistas.facecla.com.br/index.php/recadm/

Todos los pronósticos (estadístico y redes neuronales) fueron actualizados mensualmente. Es decir que para el mes de Enero de 2002, correspondiente al primer pronóstico, se utilizaron los datos de Noviembre y Diciembre del 2001. Para el siguiente pronóstico, mes de Febrero de 2002, se utilizaron los datos históricos de Noviembre, Diciembre y Enero. Para los meses siguientes se procedió de la misma manera.

\subsection{Descripción de los resultados obtenidos:}

Los resultados de los pronósticos obtenidos figuran detalladamente en el Apéndice B. Allí se puede observar una tabla con los datos obtenidos para cada producto (siete tablas en total). Cada tabla tiene los siguientes datos: Ventas históricas, el pronóstico estadístico y cuatro pronósticos de redes neuronales (para las topologías 1, 2, 3 y 4 respectivamente).

A continuación se presentan gráficamente los pronósticos obtenidos para cada serie de tiempo. Para cada una de ellas se presentan los siguientes resultados:

Gráfico 1, Datos históricos Vs. pronóstico estadístico;

Grafico 2, Datos históricos Vs. pronóstico de redes neuronales. Con respecto al pronóstico de redes neuronales, se graficó solamente una topología por producto.

\subsubsection{Resultados Serie I:}

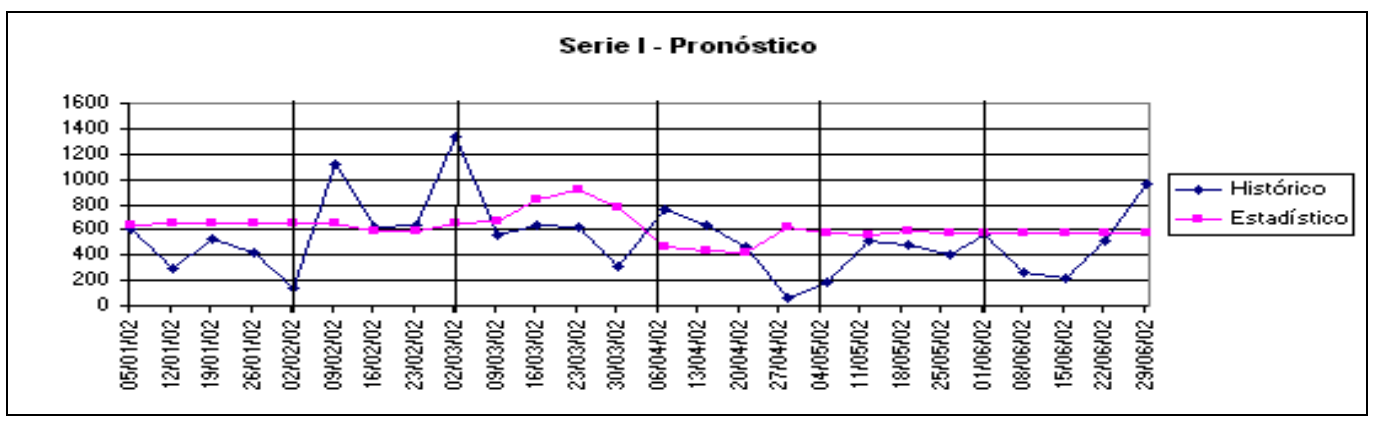

Gráfico 1 - Datos históricos y pronóstico estadístico

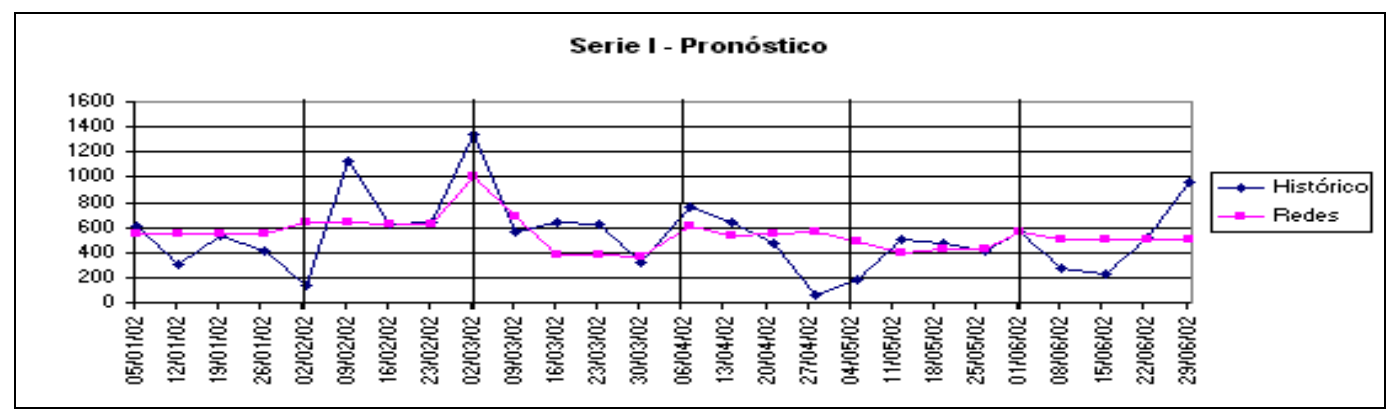

Gráfico 2 - Datos históricos y pronóstico de Redes (topología 2) 
Revista Eletrônica de Ciência Administrativa (RECADM) - ISSN 1677-7387

Faculdade Cenecista de Campo Largo - Coordenação do Curso de Administração

v. 2, n. 1, maio/2003 - http://revistas.facecla.com.br/index.php/recadm/

\subsubsection{Resultados Serie II:}

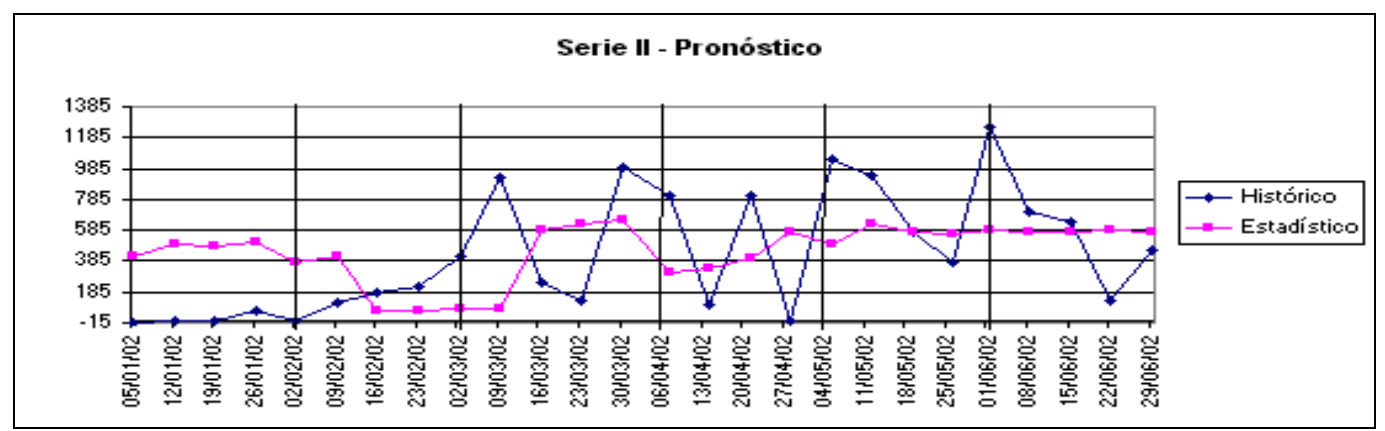

Gráfico 3 - Datos históricos y pronóstico estadístico

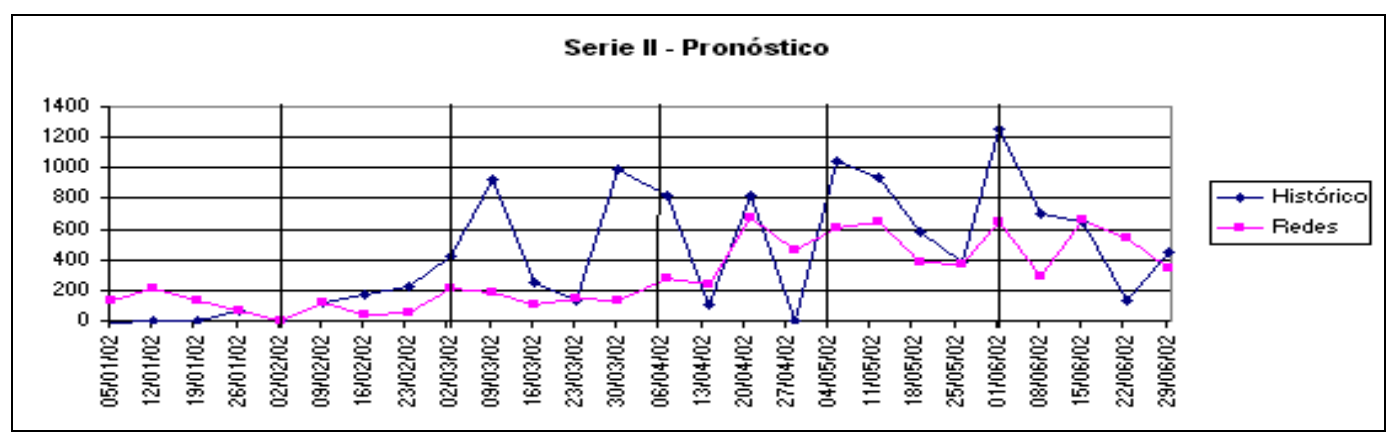

Gráfico 4 - Datos históricos y pronóstico de Redes (topología 4)

\subsubsection{Resultados Serie III:}

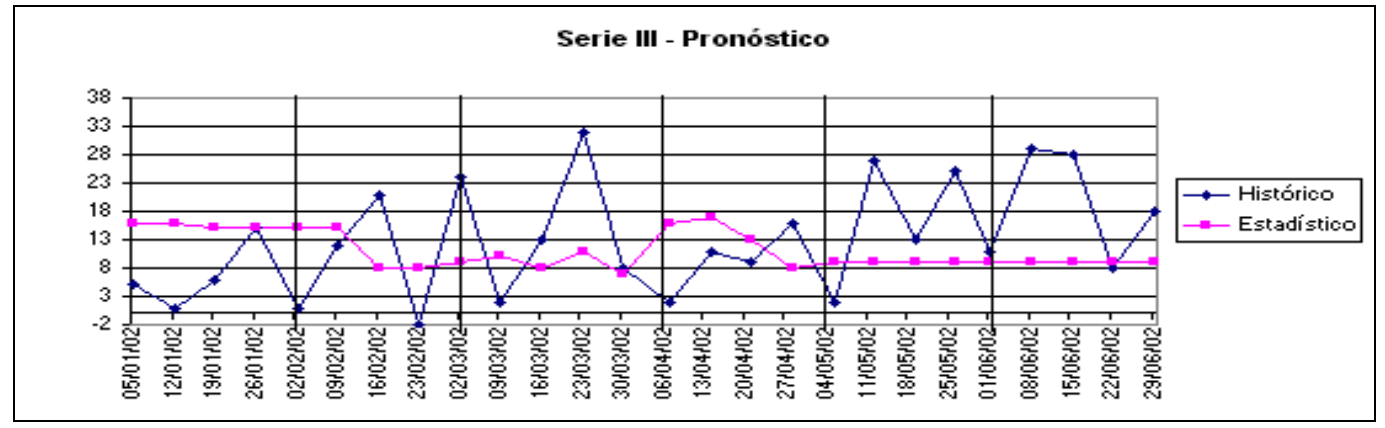

Gráfico 5 - Datos históricos y pronóstico estadístico

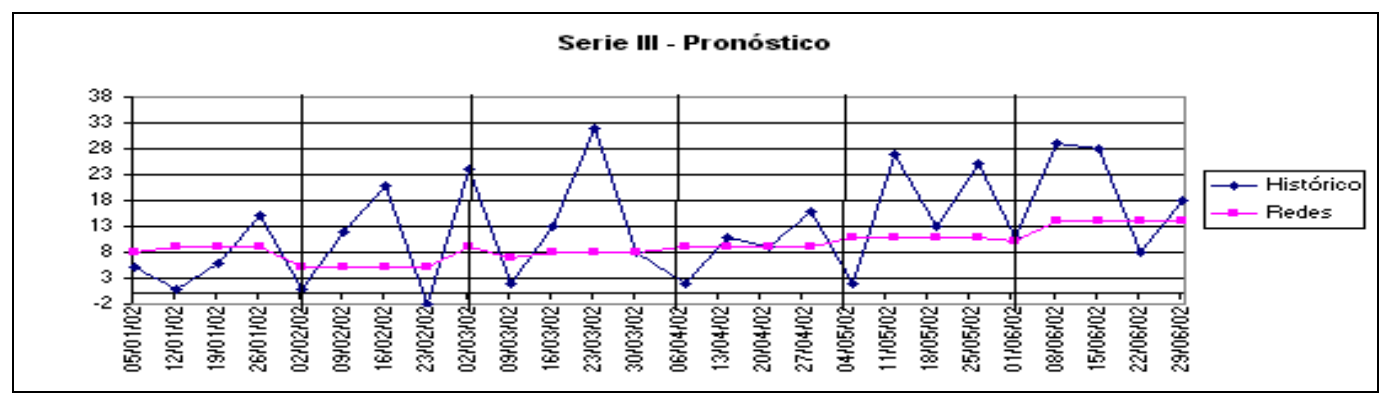

Gráfico 6 - Datos históricos y pronóstico de Redes (topología 1) 
Revista Eletrônica de Ciência Administrativa (RECADM) - ISSN 1677-7387

Faculdade Cenecista de Campo Largo - Coordenação do Curso de Administração

v. 2, n. 1, maio/2003 - http://revistas.facecla.com.br/index.php/recadm/

\subsubsection{Resultados Serie IV:}

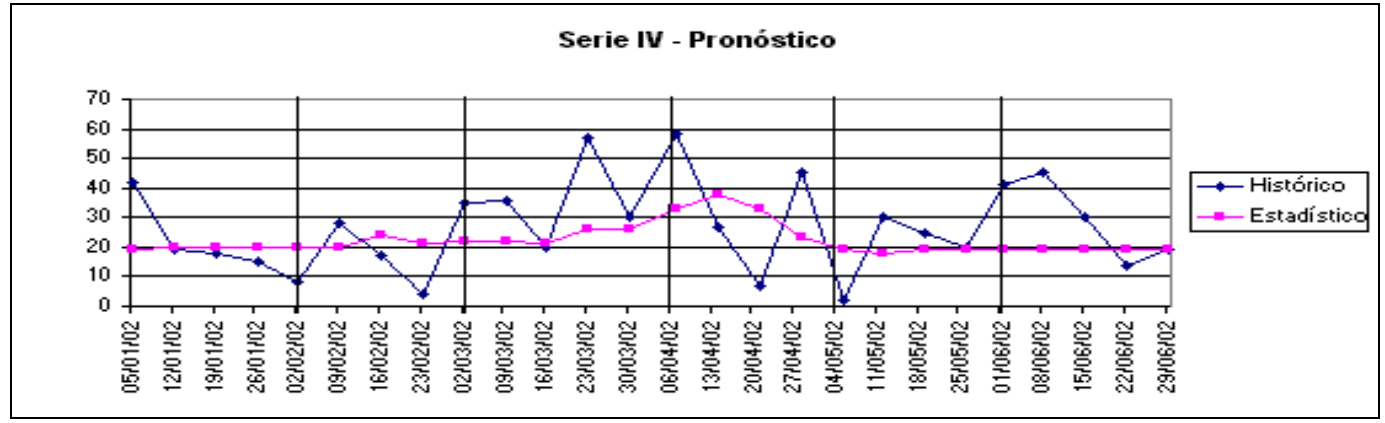

Gráfico 7 - Datos históricos y pronóstico estadístico

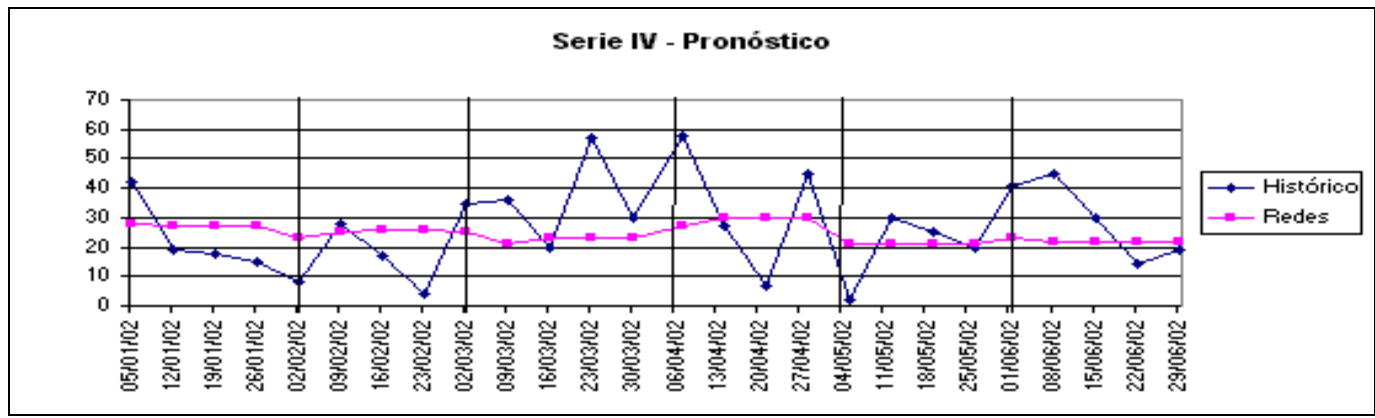

Gráfico 8 - Datos históricos y pronóstico de Redes (topología 1)

\subsubsection{Resultados Serie V:}

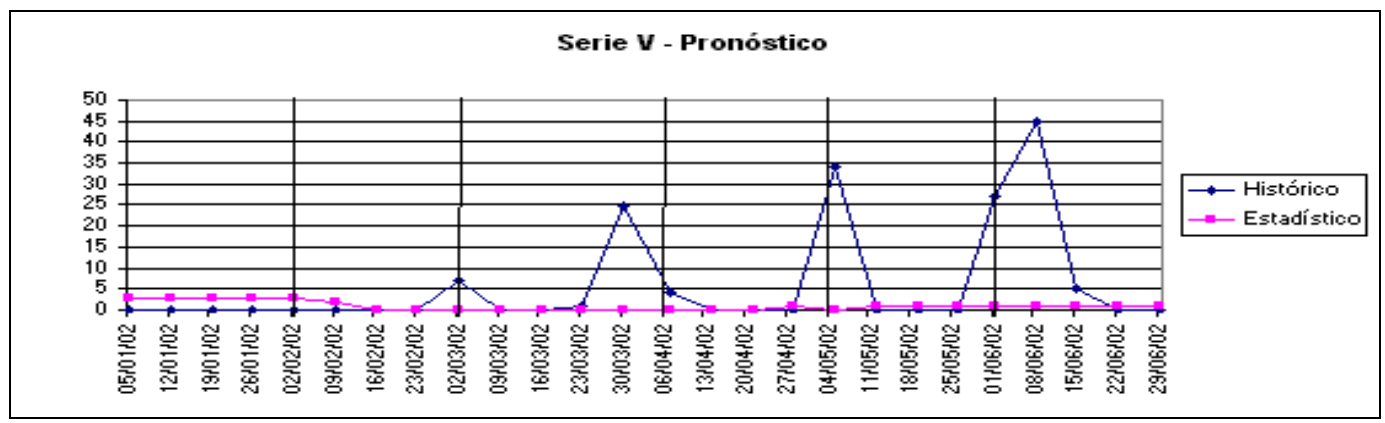

Gráfico 9 - Datos históricos y pronóstico estadístico

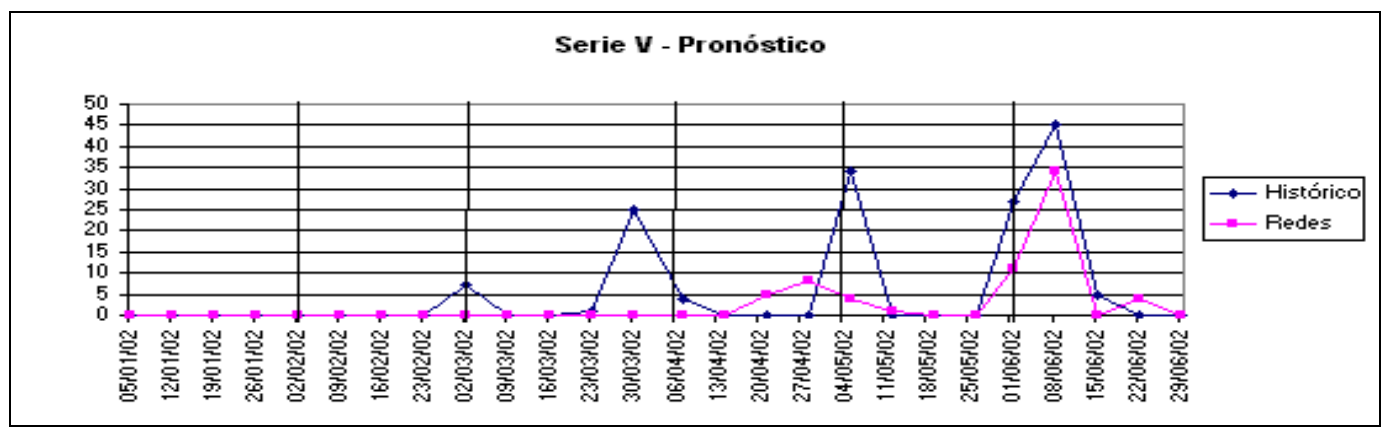

Gráfico 10 - Datos históricos y pronóstico de Redes (topología 4) 
Revista Eletrônica de Ciência Administrativa (RECADM) - ISSN 1677-7387

Faculdade Cenecista de Campo Largo - Coordenação do Curso de Administração

v. 2, n. 1, maio/2003 - http://revistas.facecla.com.br/index.php/recadm/

\subsubsection{Resultados Serie Vl:}

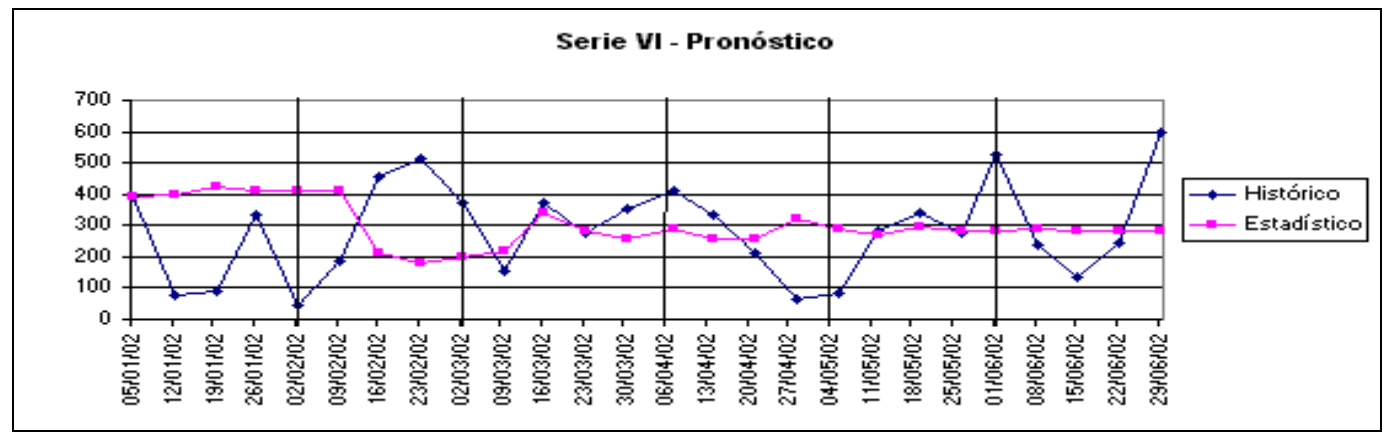

Gráfico 11 - Datos históricos y pronóstico estadístico

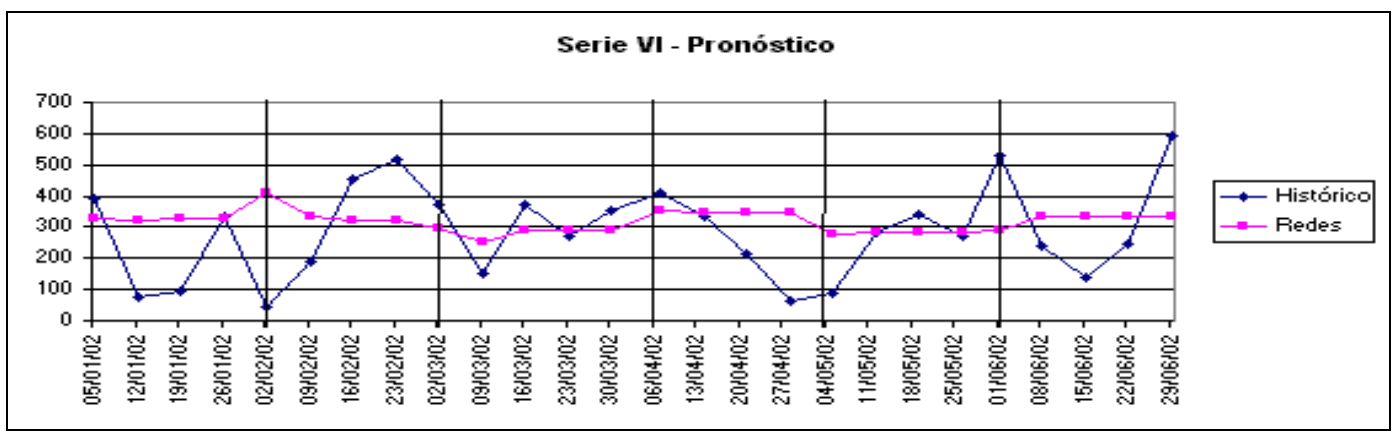

Gráfico 12 - Datos históricos y pronóstico de Redes (topología 1)

\subsubsection{Resultados Serie VII:}

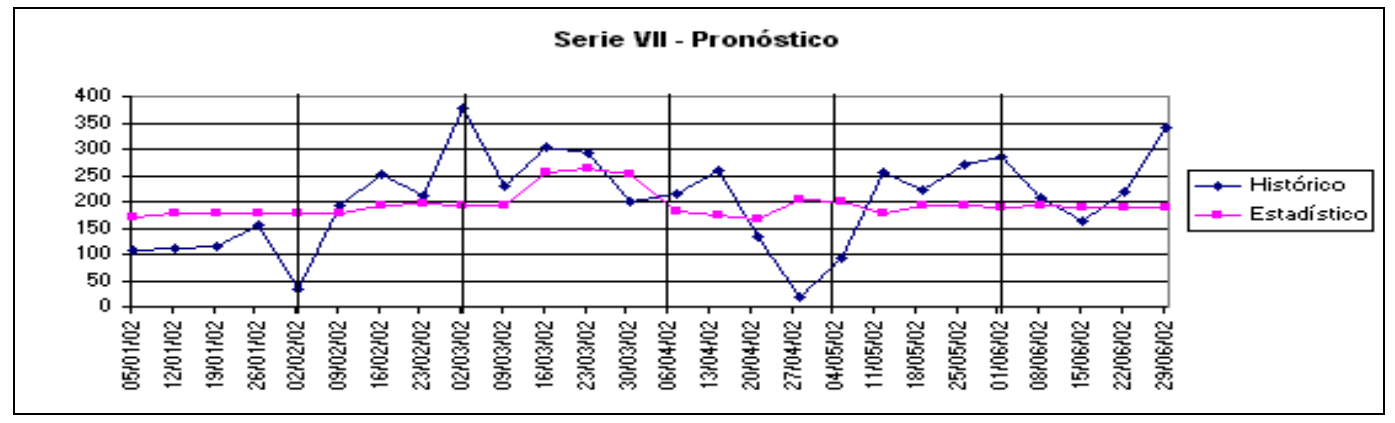

Gráfico 13 - Datos históricos y pronóstico estadístico

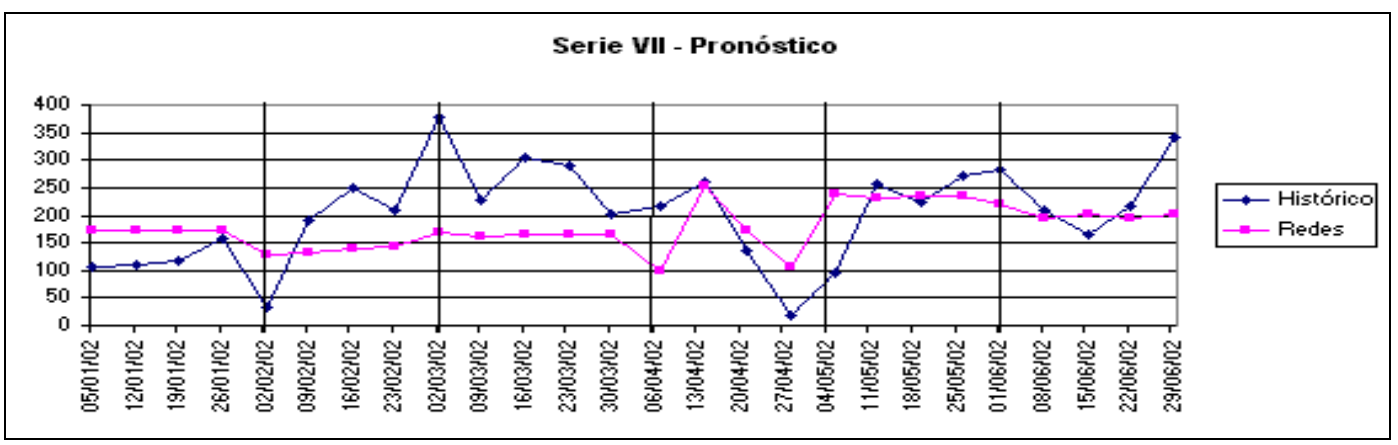

Gráfico 14 - Datos históricos y pronóstico de Redes (topología 2) 
Revista Eletrônica de Ciência Administrativa (RECADM) - ISSN 1677-7387

Faculdade Cenecista de Campo Largo - Coordenação do Curso de Administração

v. 2, n. 1, maio/2003 - http://revistas.facecla.com.br/index.php/recadm/

\subsection{La prueba de los signos de Kendall:}

Cuando la población o poblaciones fundamentales no son normales es adecuado utilizar la prueba del signo para determinar la significación de la diferencia entre dos muestras [Chambers et al., 1997; Ledesma, 1980]. Aceptando la hipótesis Cero $\left(H_{0}\right)$, la diferencia entre las medias es cero, es decir que la diferencia entre los valores menores y mayores a la media es cero, esto es que las diferencias $+\mathrm{y}-$ de los pares es cero. El objetivo de esta prueba consiste básicamente en determinar el nivel de confianza con el que podemos decir que un método es mejor que el método alternativo (Redes neuronales vs método estadístico).

\subsubsection{Procedimiento:}

La prueba del signo es un procedimiento para la probar hipótesis acerca de la media de una distribución continua.. Es una prueba no paramétrica porque no entran en juego los parámetros, sea porque no se los conoce o simplemente porque no se los utiliza. La técnica consiste en:

1. Obtener las " $n$ " diferencias di. Se obtiene calculando: $A_{i}-B_{i}=d_{i}$

La muestra A representa a la muestra del error absoluto entre los datos históricos y el pronóstico estadístico. Se calculó de la siguiente manera: Valor Absoluto (Histórico - Estadístico).

La muestra B representa a la muestra del error absoluto entre los datos históricos y el pronóstico estadístico. Se calculó de la siguiente manera: Valor Absoluto (Histórico - Redes Neuronales). Para cada serie de tiempo se calcularon 4 (cuatro) series B, cada una de ellas correspondiente a una topología de red distinta.

2. Desechar las diferencias nulas.

3. Contar el número de diferencias menores que cero. Se denomina $\mathrm{N}-$ a este valor.

4. El porcentaje de diferencias menores que cero se denomina como " $v<\mathrm{V}$ ".

5. Con el valor de $\mathrm{N}$ - se ingresa en una tabla de límites de confianza de la mediana y se calcula el nivel de confianza ("N.C") con el cual podemos asegurar que el pronóstico de redes fue más preciso que el pronóstico estadístico.

\subsubsection{Resultados de la prueba:}

A continuación se presentan los resultados obtenidos luego de realizar la prueba del signo a todos los pronósticos de cada serie. Gráficamente se presentan las muestras A (error absoluto estadístico) y B (error absoluto correspondiente a una topología determinada) para cada uno de los productos pronosticados. 
Revista Eletrônica de Ciência Administrativa (RECADM) - ISSN 1677-7387

Faculdade Cenecista de Campo Largo - Coordenação do Curso de Administração

v. 2, n. 1, maio/2003 - http://revistas.facecla.com.br/index.php/recadm/

\section{Serie l:}

\begin{tabular}{|l|c|c|c|}
\cline { 2 - 4 } \multicolumn{1}{c|}{} & V $<$ V & z & Nivel de Confianza \\
\hline Estadístico Vs. Topología 1 & 32,00 & 1,60 & $94,52 \%$ \\
\hline Estadístico Vs. Topología 2 & $\mathbf{2 6 , 9 2}$ & $\mathbf{2 , 1 6}$ & $\mathbf{9 8 , 4 5 \%}$ \\
\hline Estadístico Vs. Topología 3 & 42,31 & 0,59 & $72,19 \%$ \\
\hline Estadístico Vs. Topología 4 & 57,69 & 0,59 & $27,81 \%$ \\
\hline
\end{tabular}

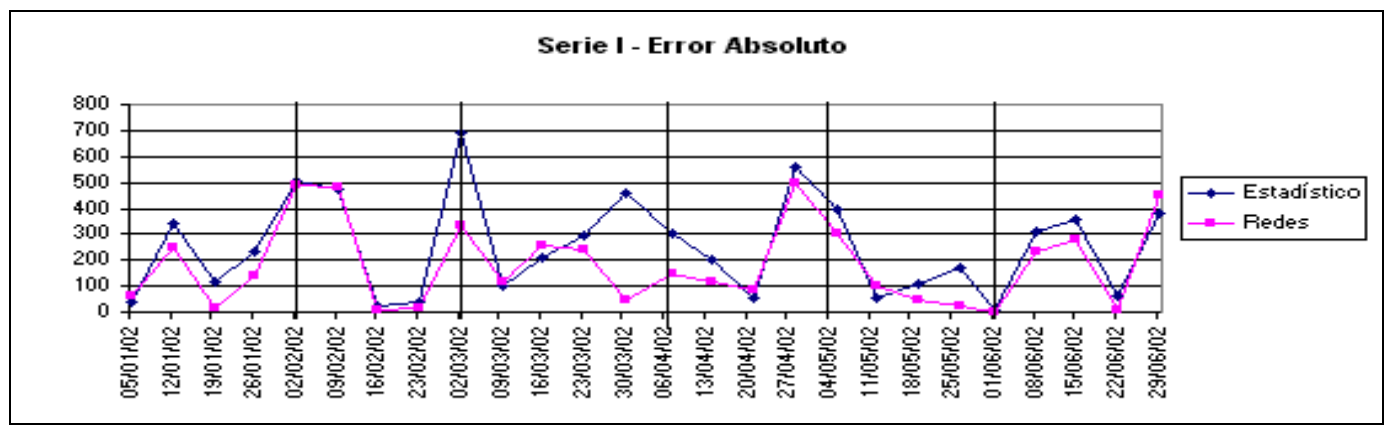

Gráfico 15 - Error Absoluto estadístico y redes neuronales (Topología 2)

Serie II:

\begin{tabular}{|l|c|c|c|}
\cline { 2 - 4 } \multicolumn{1}{c|}{} & V $<$ V & z & Nivel de Confianza \\
\hline Estadístico Vs. Topología 1 & 23,08 & 2,55 & $99,46 \%$ \\
\hline Estadístico Vs. Topología 2 & 23,08 & 2,55 & $99,46 \%$ \\
\hline Estadístico Vs. Topología 3 & 26,92 & 2,16 & $98,45 \%$ \\
\hline Estadístico Vs. Topología 4 & $\mathbf{2 3 , 0 8}$ & $\mathbf{2 , 5 5}$ & $\mathbf{9 9 , 4 6 \%}$ \\
\hline
\end{tabular}

Resultados Serie 2

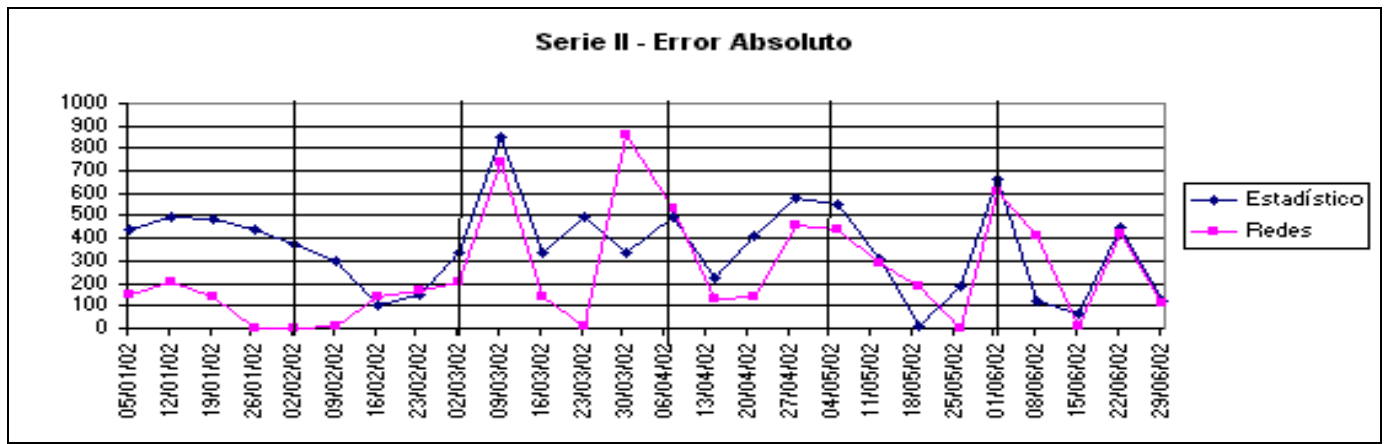

Gráfico 16 - Error Absoluto estadístico y redes neuronales (Topología 4)

Serie III:

\begin{tabular}{|l|c|c|c|}
\cline { 2 - 4 } \multicolumn{1}{c|}{} & $\mathrm{V}<\mathrm{V}$ & $\mathrm{z}$ & Nivel de Confianza \\
\hline Estadístico Vs. Topología 1 & $\mathbf{2 5 , 0 0}$ & $\mathbf{2 , 2 5}$ & $\mathbf{9 8 , 7 6 \%}$ \\
\hline Estadístico Vs. Topología 2 & 37,50 & 1,02 & $84,63 \%$ \\
\hline Estadístico Vs. Topología 3 & 34,78 & 1,25 & $89,45 \%$ \\
\hline Estadístico Vs. Topología 4 & 54,17 & 0,20 & $41,91 \%$ \\
\hline
\end{tabular}

Resultados Serie 3 
Revista Eletrônica de Ciência Administrativa (RECADM) - ISSN 1677-7387

Faculdade Cenecista de Campo Largo - Coordenação do Curso de Administração

v. 2, n. 1, maio/2003 - http://revistas.facecla.com.br/index.php/recadm/

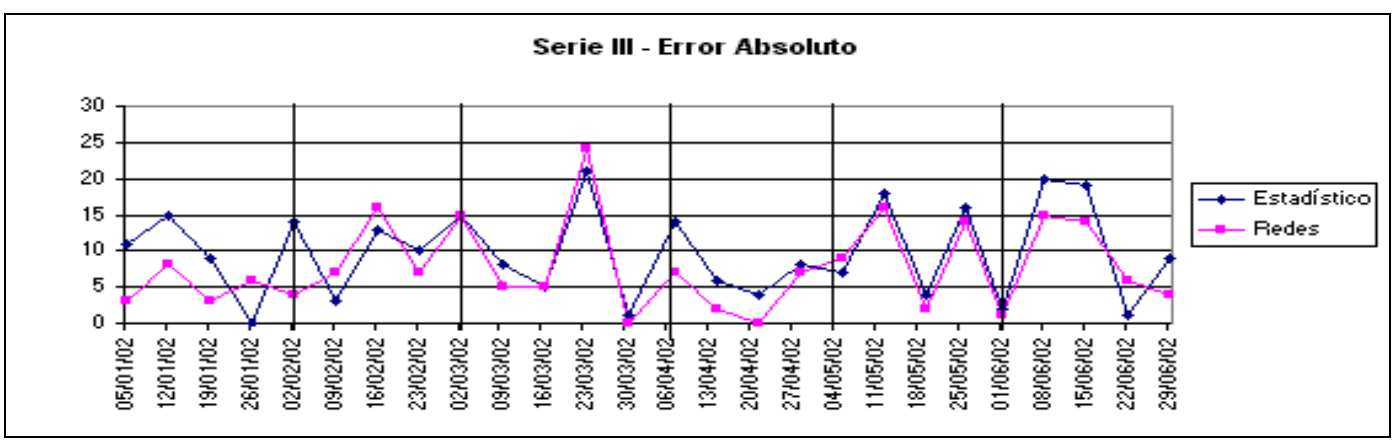

Gráfico 17 - Error Absoluto estadístico y redes neuronales (Topología 1)

\section{Serie IV:}

\begin{tabular}{|l|c|c|c|}
\cline { 2 - 4 } \multicolumn{1}{c|}{} & $\mathrm{V}<\mathrm{V}$ & $\mathrm{z}$ & Nivel de Confianza \\
\hline Estadístico Vs. Topología 1 & 56,00 & 0,40 & $34,46 \%$ \\
\hline Estadístico Vs. Topología 2 & $\mathbf{5 4 , 1 7}$ & $\mathbf{0 , 2 0}$ & $\mathbf{4 1 , 9 1 \%}$ \\
\hline Estadístico Vs. Topología 3 & 65,38 & 1,37 & $8,49 \%$ \\
\hline Estadístico Vs. Topología 4 & 65,38 & 1,37 & $8,49 \%$ \\
\hline
\end{tabular}

\section{Resultados Serie 4}

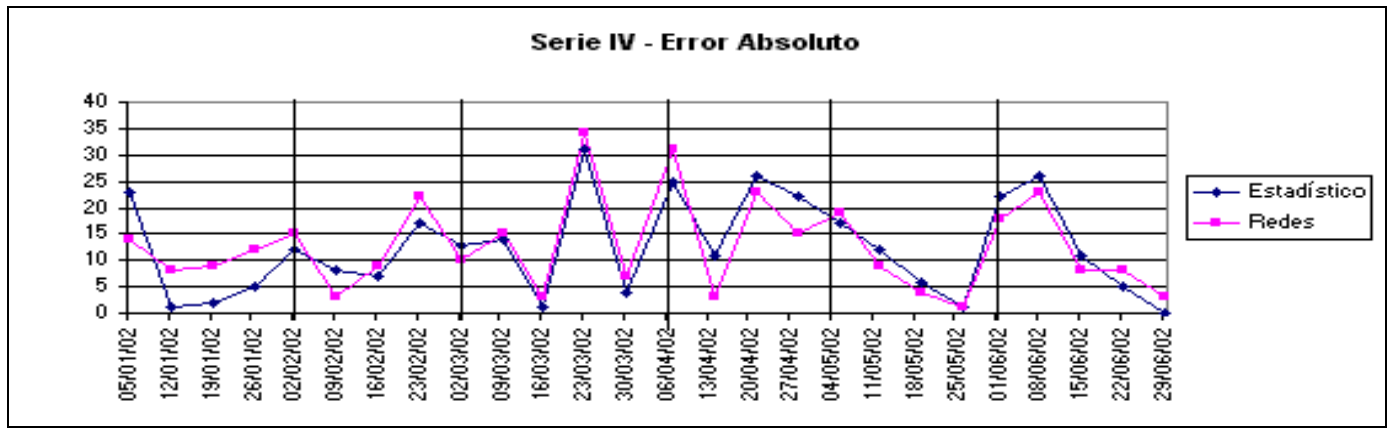

Gráfico 18 - Error Absoluto estadístico y redes neuronales (Topología 2)

Serie V:

\begin{tabular}{|l|c|c|c|}
\cline { 2 - 4 } \multicolumn{1}{c|}{} & $\mathrm{V}<\mathrm{V}$ & $\mathrm{z}$ & Nivel de Confianza \\
\hline Estadístico Vs. Topología 1 & 56,52 & 0,42 & $33,83 \%$ \\
\hline Estadístico Vs. Topología 2 & 50,00 & $-0,25$ & $40,13 \%$ \\
\hline Estadístico Vs. Topología 3 & 47,37 & 0,00 & $50,00 \%$ \\
\hline Estadístico Vs. Topología 4 & $\mathbf{2 5 , 0 0}$ & $\mathbf{1 , 7 5}$ & $\mathbf{9 5 , 9 9 \%}$ \\
\hline
\end{tabular}

Resultados Serie 5



Gráfico 19 - Error Absoluto estadístico y redes neuronales (Topología 4) 
Revista Eletrônica de Ciência Administrativa (RECADM) - ISSN 1677-7387

Faculdade Cenecista de Campo Largo - Coordenação do Curso de Administração

v. 2, n. 1, maio/2003 - http://revistas.facecla.com.br/index.php/recadm/

Serie VI:

\begin{tabular}{|l|c|c|c|}
\cline { 2 - 4 } \multicolumn{1}{c|}{} & $\mathrm{v}<\mathrm{V}$ & $\mathrm{z}$ & Nivel de Confianza \\
\hline Estadístico Vs. Topología 1 & $\mathbf{3 8 , 4 6}$ & $\mathbf{0 , 9 8}$ & $\mathbf{8 3 , 6 6 \%}$ \\
\hline Estadístico Vs. Topología 2 & $\mathbf{4 6 , 1 5}$ & 0,20 & $57,77 \%$ \\
\hline Estadístico Vs. Topología 3 & 42,31 & 0,59 & $72,19 \%$ \\
\hline Estadístico Vs. Topología 4 & 50,00 & $-0,20$ & $42,23 \%$ \\
\hline
\end{tabular}

Resultados Serie 6

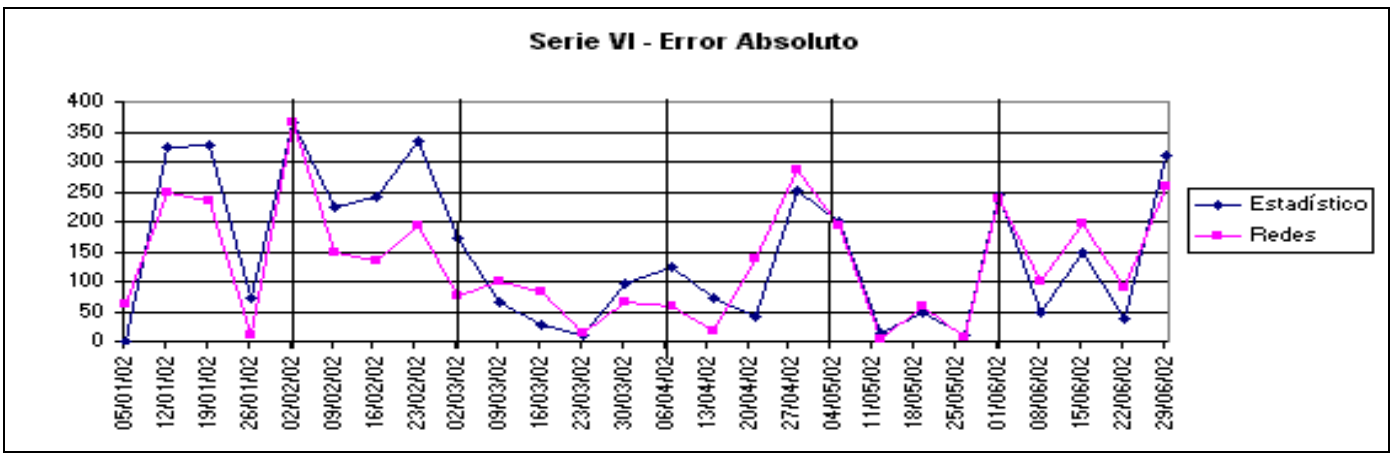

Gráfico 20 - Error Absoluto estadístico y redes neuronales (Topología 1)

Serie VII:

\begin{tabular}{|l|c|c|c|}
\cline { 2 - 4 } \multicolumn{1}{c|}{} & $\mathrm{v}<\mathrm{V}$ & $\mathrm{z}$ & Nivel de Confianza \\
\hline Estadístico Vs. Topología 1 & $\mathbf{7 6 , 9 2}$ & $\mathbf{2 , 5 5}$ & $\mathbf{0 , 5 4 \%}$ \\
\hline Estadístico Vs. Topología 2 & $\mathbf{4 6 , 1 5}$ & $\mathbf{0 , 2 0}$ & $\mathbf{5 7 , 7 7 \%}$ \\
\hline Estadístico Vs. Topología 3 & 57,69 & 0,59 & $\mathbf{2 7 , 8 1 \%}$ \\
\hline Estadístico Vs. Topología 4 & 57,69 & 0,59 & $\mathbf{2 7 , 8 1 \%}$ \\
\hline
\end{tabular}

Resultados Serie 7

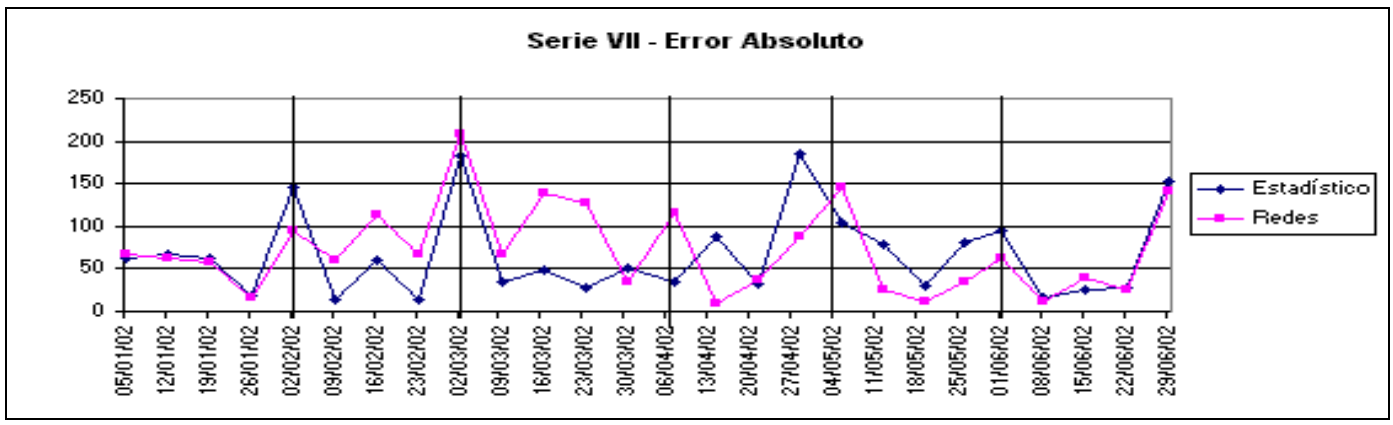

Gráfico 21 - Error Absoluto estadístico y redes neuronales (Topología 2)

\section{Conclusiones:}

\section{Conclusión 1:}

Los resultados nos permiten concluir con un nivel de confianza del $82.29 \%$ que las redes neuronales realizaron un pronóstico más acertado en comparación al método estadístico utilizado. Este resultado corresponde al promedio de los mejores pronósticos para cada serie de tiempo.

\begin{tabular}{|l|c|c|c|c|c|c|c|c|}
\hline Mejor Resultado & $\mathbf{9 8 , 4 5 \%}$ & $\mathbf{9 9 , 4 6 \%}$ & $\mathbf{9 8 , 7 6} \%$ & $\mathbf{4 1 , 9 1 \%}$ & $\mathbf{9 5 , 9 9}$ & $\mathbf{8 3 , 6 6 \%}$ & $\mathbf{5 7 , 7 7 \%}$ & $\mathbf{8 2 , 2 9} \%$ \\
\hline Mejores 2 resultados & $96,49 \%$ & $99,46 \%$ & $94,11 \%$ & $38,19 \%$ & $\mathbf{7 3 , 0 0} \%$ & $\mathbf{7 7 , 9 2 \%}$ & $\mathbf{4 2 , 7 9 \%}$ & $\mathbf{7 4 , 5 6 \%}$ \\
\hline Peor Resultado & $27,81 \%$ & $98,45 \%$ & $41,91 \%$ & $8,49 \%$ & $33,83 \%$ & $42,23 \%$ & $0,54 \%$ & $36,18 \%$ \\
\hline Promedio de Serie & $73,24 \%$ & $99,21 \%$ & $78,69 \%$ & $23,34 \%$ & $54,99 \%$ & $63,96 \%$ & $28,49 \%$ & $60,27 \%$ \\
\hline
\end{tabular}


Revista Eletrônica de Ciência Administrativa (RECADM) - ISSN 1677-7387

Faculdade Cenecista de Campo Largo - Coordenação do Curso de Administração

v. 2, n. 1, maio/2003 - http://revistas.facecla.com.br/index.php/recadm/

Uno de los cuadros que resultó más interesante para analizar fue el Cuadro 3. En el mismo están indicados con la letras $C$ los casos en donde el error absoluto del pronóstico de Redes fue mayor que el error absoluto del pronóstico estadístico. Los casos A y B representan los pronósticos que tuvieron menor error absoluto (A representa el mejor de los pronósticos para cada serie).

\begin{tabular}{|c|c|c|c|c|c|c|c|c|}
\hline \multicolumn{2}{|c|}{} & Serie I & Serie II & Serie III & Serie IV & Serie V & Serie VI & Serie VII \\
\hline \multirow{2}{*}{$\frac{}{\circ}$} & I & B & B & A & C & C & A & C \\
\cline { 2 - 9 } & II & A & B & B & C & B & B & C \\
\cline { 2 - 9 } & III & B & B & B & C & B & B & C \\
\cline { 2 - 9 } & IV & B & A & B & C & A & B & C \\
\hline
\end{tabular}

Cuadro 3 - Resultados generales

Del cuadro 2 y el cuadro 3 (los dos cuadros anteriores) podemos inferir que hay casos bien definidos, Serie IV y Serie VII, en donde el pronóstico de redes es claramente inferior al pronóstico estadístico. Se obtuvo un nivel de confianza promedio de $23.34 \%$ y $28.49 \%$ respectivamente. Por lo tanto podemos decir que para esos dos productos no es conveniente utilizar el pronóstico de Redes neuronales.

Si ahora analizamos los resultados del pronóstico sin tener en cuenta aquellos productos en donde claramente no conviene utilizar redes se obtiene el siguiente cuadro:

\begin{tabular}{|l|c|c|c|c|c|c|}
\cline { 2 - 7 } \multicolumn{1}{c|}{} & Serie 1 & Serie 2 & Serie 3 & Serie 5 & Serie 6 & Promedio \\
\hline Mejor Resultado & $\mathbf{9 8 , 4 5 \%}$ & $\mathbf{9 9 , 4 6 \%}$ & $\mathbf{9 8 , 7 6 \%}$ & $\mathbf{9 5 , 9 9 \%}$ & $\mathbf{8 3 , 6 6 \%}$ & $\mathbf{9 5 , 2 7 \%}$ \\
\hline Mejores 2 resultados & $96,49 \%$ & $99,46 \%$ & $94,11 \%$ & $73,00 \%$ & $77,92 \%$ & $88,19 \%$ \\
\hline Peor Resultado & $27,81 \%$ & $98,45 \%$ & $41,91 \%$ & $33,83 \%$ & $42,23 \%$ & $48,85 \%$ \\
\hline Promedio de Serie & $73,24 \%$ & $99,21 \%$ & $78,69 \%$ & $54,99 \%$ & $63,96 \%$ & $74,02 \%$ \\
\hline
\end{tabular}

Cuadro 4 - Nivel de confianza

Los resultados del cuadro anterior, nos permiten decir que el nuevo enfoque para realizar pronósticos es superior al enfoque estadístico con un nivel de confianza promedio del $95.27 \%$.

\section{Conclusión 2:}

En la mayoría de los casos no hubo una topología determinada que tenga un rendimiento superior a las demás. El promedio del nivel de confianza para cada topología nos indica que para cada producto habría una topología determina con la cual podríamos obtener pronósticos más precisos.

\begin{tabular}{|l|c|c|}
\cline { 2 - 3 } \multicolumn{1}{c|}{} & Mejor Resultado & Promedio Total \\
\hline Topología 1 & $99,46 \%$ & $63,60 \%$ \\
\hline Topología 2 & $99,46 \%$ & $68,59 \%$ \\
\hline Topología 3 & $98,45 \%$ & $59,80 \%$ \\
\hline Topología 4 & $99,46 \%$ & $49,10 \%$ \\
\hline \multicolumn{2}{|c|}{ Cuadro 5 - Topologías }
\end{tabular}


Revista Eletrônica de Ciência Administrativa (RECADM) - ISSN 1677-7387

Faculdade Cenecista de Campo Largo - Coordenação do Curso de Administração

v. 2, n. 1, maio/2003 - http://revistas.facecla.com.br/index.php/recadm/

\section{Conclusión 3:}

En cuanto al conocimiento necesario para realizar los pronósticos estadísticamente o mediante la utilización de redes neuronales podemos decir que es menor en el caso de las redes neuronales. Sin embargo, el usuario necesita saber qué topología y método de entrenamiento debe aplicar para cada caso en particular.

\section{Futuras líneas de trabajo:}

De las conclusiones anteriores se desprende la necesidad de trabajar en ciertas líneas de trabajo:

(a) Realizar una clasificación de las series de tiempo (o productos) para poder determinar cuando conviene utilizar el método estadístico o el nuevo enfoque de redes neuronales. Se podría utilizar una técnica de Data mining.

(b) Para aquellos casos en los que sea conveniente utilizar el enfoque de redes neuronales se debería poder determinar qué topología utilizar.

(c) Analizar cómo afecta al rendimiento de los métodos analizados la cantidad de datos que se utilicen como entrada. Analizar si a medida que incluyo mayor cantidad de datos cambia el rendimiento de los métodos utilizados.

(d) En cuanto a la comercialización de productos software, la misma ha sido y sigue siendo la forma más extendida para simular redes neuronales. La diferencia entre los distintos productos software radica en aspectos tales como el tipo y número de arquitecturas de red que soporta, velocidad de procesamiento, interfaz gráfica, etc.

Se debería tener en cuenta la posibilidad de desarrollar una herramienta que utilice redes neuronales para realizar pronósticos. La misma podría trabajar en conjunto con los métodos estadísticos. Por lo tanto, un aspecto claves a tener en cuenta sería el desarrollo del:

Aprendizaje sin supervisión. La aplicación que se desarrolle debería prescindir del supervisor para elegir la arquitectura adecuada, realizar el entrenamiento y finalmente, pronosticar las ventas.

\section{Referencias:}

Chambers, J. Mullick, S. Y Smith, D. 1997. Cómo elegir la técnica de pronóstico correcta. Biblioteca Harvard. España.

Derevitsiotis, K. 1981. Operations Management. Mc Graw-Hill. EE.UU.

Devore, J. 1998. Probabilidad y estadística para ingeniería y ciencias. Thompson Editores. EE.UU.

Hilera, J. Y Martínez, V. 1995. Redes Neuronales Artificiales: Fundamentos, modelos y aplicaciones. Ra-Ma. España.

IBM. 1994.Neural Network Utility Versión 3.1: Installation and user's guide. IBM Press. EE.UU.

Kazmier, L. 1999. Estadística aplicada a la administración y la economía. Mc Graw-Hill. EE.UU Ledesma, D. 1980. Estadística médica. Editorial Universitaria de Buenos Aires. Argentina.

Sapag C. 2000. Preparación y evaluación de proyectos. Mc-Graw Hill. Mexico.

SRM. 1996. Manual del software Supplier Relationship Management. Appendix A: Forecast Calculation Methods. Rdwards Inc. EE.UU.

Sitio de Internet: http://ubmail.ubalt.edu/ harsham/stat-data/opre330Forecast.htm 